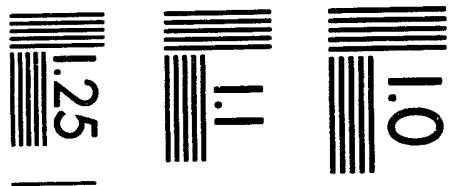

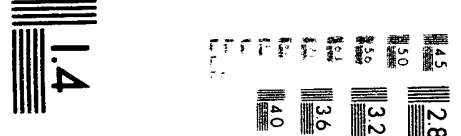

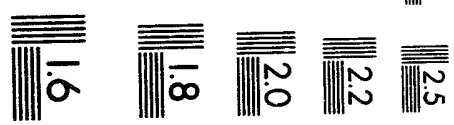



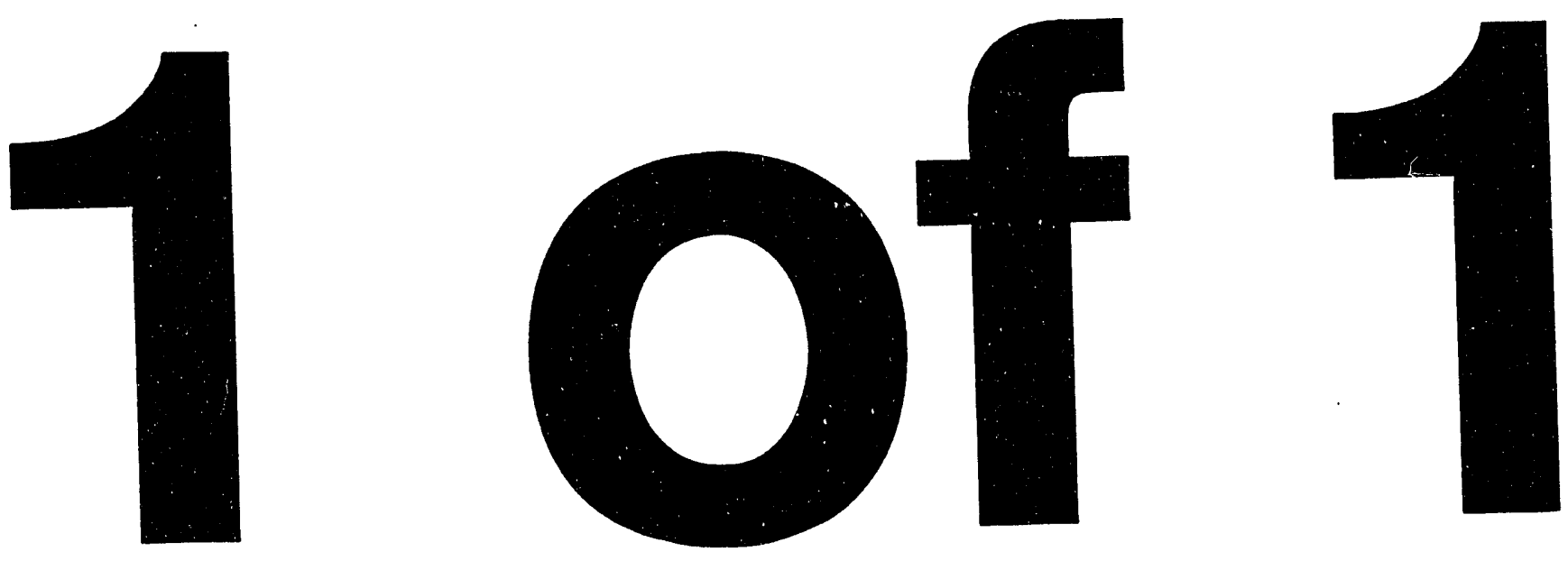


\title{
ON THE ORIGIN OF PORPHYRITIC CHONDRULES
}

\author{
Milton Blander ${ }^{1}$, Laura Unger ${ }^{2}$, Arthur Pelton ${ }^{3}$ and Gunnar Ericksson ${ }^{3}$
}

\author{
${ }^{1}$ Argonne National Laboratory \\ 9700 South Cass Avenue \\ Argonne, IL 60439
}

\section{${ }^{2}$ Purdue University-North Central, Westville, In 46391}

\author{
${ }^{3}$ Ecole Polytechnique, \\ Montreal, Quebec, Canada H3C 3A7 \\ DISCLAIMER
}

\begin{abstract}
This report was prepared as an account of work sponsored by an agency of the United States Government. Neither the United States Government nor any agency thereof, nor any of their employees, makes any warranty, express or implied, or assumes any legal liability or responsibility for the accuracy, completeness, or usefulness of any information, apparatus, product, or process disclosed, or represents that its use would not infringe privately owned rights. Reference herein to any specific commercial product, process, or service by trade name, trademark, manufacturer, or otherwise does not necessarily constitute or imply its endorsement, recommendation, or favoring by the United States Government or any agency thereof. The views and opinions of authors expressed herein do not necessarily state or reflect those of the United States Government or any agency thereof.
\end{abstract}

The submitted manuscript has been authored
by a contractor of the U.S. Government
Under contract No. W.31-109ENG.38.
Accordingly, the U.S. Government retains a
nonexclusive, royalty.free license to publish
or reproduce the published form of this
contribution, or allow others to do $\$$, for
$U$.S. Government purposes.

This work was supported by the U. S. Department of Energy, Division of Materials Sciences, Office of Basic Energy Sciences under contract No. W-31-109-ENG-38. 


\title{
On the Origin of Porphyritic Chondrules
}

\author{
Milton Blander ${ }^{1}$, Laura Unger ${ }^{2}$, Arthur Pelton ${ }^{3}$ and Gunnar Ericksson ${ }^{3}$ \\ ${ }^{1}$ Argonne National Laboratory, Argonne, IL 60439-4837 \\ ${ }^{2}$ Purdue University-North Central, Westville, IN 46391 \\ ${ }^{3}$ Ecole Polytechnique, Montreal, Quebec, Canada H3C 3A7
}

Abstract

A unique computer program for the calculation of the complex equilibria in a cooling nebular gas has been used to explore a possible origin of porphyritic chondrules, (the major class of chondrules in chondritic meteorites) as well as other classes of chondrules. A keystone of the calculation is a method of accurately calculating the thermodynamic properties of molten multicomponent aluminosilicates which allows one to deduce the compositions of silicate condensates as functions of temperature and pressure of a nebular gas. This computer program is coupled with a chemical equilibrium algorithm for the calculation of complex equilibria in multicomponent-multiphase systems with at least 1000 chemical species and with a data base of over 5000 solid, liquid and gaseous species.

In addition, we have considered the effect of two major predicted nucleation constraints on the formation of porphyritic chondrules; these are (a) a large barrier to the formation of condensed iron alloys which leads to the supersaturation of iron in the vapor. This results in the incorporation of much more iron oxide in the silicates than one has at equilibrium and (b) a barrier to the condensation of solid silicates which leads to the condensation of metastable subcooled liquid silicates which ultimately form chondrules.

Constrained equilibrium calculations for a gas containing $\mathrm{H}, \mathrm{C}, \mathrm{O}, \mathrm{Si}, \mathrm{S}, \mathrm{Fe}, \mathrm{Ca}$, $\mathrm{Na}, \mathrm{Al}$ and $\mathrm{Mg}$ in solar proportions indicate that types IA and II porphyritic chondrules 
could have condensed at pressures ranging from about $10^{-2}$ to 1 atmospheres with compositions resembling the low FeO type IA Semarkona chondrules forming a temperatures with are $119^{\circ}$ to $157^{\circ}$ higher than compositions resembling the high $\mathrm{FeO}$ type II Semarkona chondrules. It is postulated that crystallization of the metastable liquids after spontaneous nucleation or nucleation induced by nebular perturbations, freezes in these compositions. Droplets which do not nucleate to crystallize to type IA or II chondrules, survive to lower temperatures and will pick up more silica to be pyroxene normative and be high in iron oxide. Because pyroxene is difficult to nucleate, these droplets will tend to form type III chondrules (excentroradial pyroxene chondrules nucleated by an external seed, or glassy or cryptocrystalline chondrules).

This mode of formation of chondrules gets around an unsurmountable difficulty with current mechanisms for forming porphyritic chondrules which appear to require external heating of materials of different compositions and different locations to just below their very different liquidus temperatures, a very unlikely process. Crystallization of metastable subcooled liquids (recalescence) does just that in the first step. Recalescence and the temperature cycling expected in a convective nebula are likely to lead to the properties and textures observed in porphyritic and type III chondrules. However, experiments will be needed to test our hypotheses and to further define nebular conditions which can produce these chondrule types.

The most important aspects of our results are that (1) the different types of chondrules (types I, II, III) could have been produced from the same (solar) gas and do not need a different gas for each apparent oxidation state and (2) the overwhelming 
difficulty of "just so" heating of spherules in current models for making porphyritic chondrules is eliminated. 
Introduction

Chondritic meteorites provide important clues on the origins of condensed matter in the solar system. The presence of non-equilibrium effects in chondrites and in the chondrules they contain ${ }^{1-5}$ greatly enhances the information content and the number of clues locked in chondrite properties. In this paper we examine possible non-equilibrium effects on the origin of chondrules which had led to the freezing in of chondrule compositions and textures. In ordinary unequilibrated chondrites there is a spectrum of chondrule compositions which defies a simple explanation. In this paper we will use a simple example of differences in chondrule properties to illustrate a new approach which we hope will ultimately provide a unified explanation of the mode of formation of chondrules.

There are different types of meteoritic chondrule classes in ordinary chondrites with different chemistries and different textures. ${ }^{6-11}$ Such differences have been thought to require different compositions of the nebular gases from which these different classes originated $^{6,12}$ with an improbable subsequent assemblage of these chondrules from different locations. For any one type of chondrite, this requires "just so" mixing in order to get the compositions for that type. In this paper, we will show that two of the most important classes of chondrules, porphyritic classes IA and II that are present in all ordinary chondrites, could have readily originated from the same gaseous environment by crystallization and freezing in of reactions with the nebula at different temperatures to be later assembled into a parent body at yet lower temperatures. We will also briefly 
discuss the origins of the other classes of chondrules from the same gas using the same concepts. Fortunately, one of these bodies, the source of the LL 3.0 chondrite Semarkona, was assembled at a temperature which was apparently low enough ( $<525$ $\mathrm{K}^{6}$ ) to preserve the record of the original compositions. (The designation 3.0 indicates the lowest degree of metamorphism (or automorphism) encountered in ordinary chondrites.)

Our study utilizes a unique computer program for the calculation of the complex equilibria in a cooling nebular gas which can handle at least 1000 chemical species in different phases including the nebular gas, liquid and solid condensates which includes a large number of separate compound phases, and solid and liquid solutions. ${ }^{13}$ Especially important in our calculations is a method for the calculation of the thermodynamic properties of molten aluminosilicates ${ }^{14-18}$ which permits us to determine the compositions of condensates as a function of temperature. In addition, we consider two kinds of expected nucleation constraints on the process of condensation ${ }^{1}$, (a) on the nucleation of metallic iron and (b) on the formation of solid silicates. The constraint (a) leads to large slipersaturations of iron vapor which raises the activity and concentrations of $\mathrm{FeO}$ in the silicates and makes the iron oxide concentration a fairly strong function of temperature. The constraint (b) leads to the direct condensation of metastable subcooled liquids (analogous to the metastable liquid water in terrestrial clouds) with the degree of subcooling needed to produce a liquid being a function of the nebular gas pressures..$^{1-4}$ Both constraints are based on well understood physical phenomena. If correct, these processes impose important constraints on the nebular temperatures and pressures.

In order to simplify the presentation, we will not discuss in detail the many different 
processes proposed by others for the formation of chondrules but will focus on our mechanism which requires a nebular environment with far simpler properties (e.g. uniform composition) that are necessary for all other chondrule forming mechanisms. We will present currently popular concepts for comparisons with our results.

We have chosen to focus largely on type IA and II chondrules because they are the most abundant ${ }^{9}$ and they are similar in texture (both are porphyritic) but have very different compositions (Table I) which, at equilibrium, can not be formed from the same gaseous environment. (Type IA is much more abundant than Type $11 .{ }^{9}$ ) The calculations for these two classes provide a simple and clear picture of the potential significance of our mechanism. An important result is that the compositions of chondrules in a nebula at a given pressure are governed by the temperature. With a nucleation constraint on iron condensation, the temperature dependence of the FeO content of chondrules is large until a major fraction of $\mathrm{Fe}$ has condensed. Thus, as is exhibited below, the FeO content correlates with the content of the relatively volatile silica. Our approach also leads to plausible mechanisms for the formation of the other types of chondrules, e.g. radial pyroxene, glassy and cryptocrystalline chondrules and barred olivine chondrules.

\section{Calculations of Condensation in a Nebula}

We consider an origin of these chondrules from a gas of solar composition ${ }^{19}$ to show that the compositions of both types of chondrules could have been produced in the same location. We used a computer program which calculates equilibria in complex systems with over 1000 species in various solid, liquid and gaseous phases. Some of the condensed phases are solutions and we include data for pyroxenes, and olivines ${ }^{20}$, 
plagioclase $^{21}$, molten aluminosilicates ${ }^{14-18}$ and the relevant data on geologic materials of Berman and Brown ${ }^{22}$ and Berman et al. ${ }^{23}$ which has been reanalyzed and modified very slightly so as to be consistent with the geologic equilibria used by these authors as well as with binary and ternary phase diagrams which include these geological materials and with the properties of the corresponding binary and ternary liquids. For these calculations we consider condensation from a gas consisting of $\mathrm{H}, \mathrm{C}, \mathrm{O}, \mathrm{Si}, \mathrm{S}, \mathrm{Fe}, \mathrm{Ca}, \mathrm{Na}, \mathrm{Al}$ and $\mathrm{Mg}$ at pressures ranging from $10^{-3}$ to 5 atmospheres.

In addition, we impose two kinetic constraints on the calculation. The first is a large nucleation constraint on the condensation of metallic iron. At equilibrium, iron should condense as a solid. However, the condensation of solid iron should be influenced by a nucleation barrier greater than that of the liquid which itself has a very large barrier to nucleation as a result of the large surface tensions (of the order of 1800 ergs $\mathrm{cm}^{-2}$ ). ${ }^{1}$ Consequently one predicts and experimentally finds very large supersaturations of iron. ${ }^{24}$ The second constraint is on the formation of solid silicates which has a larger barrier to nucleation than liquid silicates. This leads to the direct condensation of metastable subcooled liquids. Indeed, from the presence of olivines with fayalite rich cores, Jories ${ }^{7}$ deduced that type II chondrules were supercooled. In fact, the compositions of the cores, $\mathrm{Fa}_{20}$, is consistent with expectations for deeply metastable, subcooled liquids; with large subcooling, the chemical driving force for the initial stage of crystallization is so great that the crystals form rapidly; once nucleated, the composition of the rapidly crystallizing olivine is diffusion controlled so that if $\mathrm{Fe}^{2+}$ and $\mathrm{Mg}^{2+}$ have similar mobilities, one would expect that their compositional ratios would be about the same as those of the melts. 
From the data Taule 1a, we can deduce a composition of about $\mathrm{Fa}_{21}$ from the $\mathrm{Fe}^{2+} / \mathrm{Mg}^{2+}$ ratio in the average of the type $I I$ chondrule compositions in Semarkona. This provides definitive support for an origin from metastable subcooled droplets.

\section{Calculations}

Our calculations will be compared with the average bulk compositions of type la and II chondrules given by McCoy et al. ${ }^{6}$ In Table la, we present these compositions recalculated by leaving out the minor constituents (which total less than $1 \%$ of the chondrule) $\mathrm{TiO}_{2}, \mathrm{Cr}_{2} \mathrm{O}_{3}, \mathrm{MnO}$ and $\mathrm{K}_{2} \mathrm{O}$, converting to mole percent and normalizing the compositions to total 100 mole \%. As a reference point for our calculations of condensation from a solar nebula, we have calculated the equilibrium liquidus temperatures for these measured types IA and II average compositions as $2026 \mathrm{~K}$ and $1904 \mathrm{~K}$ respectively. Calculations were made for condensation from a solar nebula with a kinetic constraint blocking the formation of metallic iron and solid silicates. Consequently, the iron in the vapor becomes supersaturated and has partial pressures above the equilibrium vapor pressure and the reaction

$$
\mathrm{Fe}(\mathrm{g})+\mathrm{H}_{2} \mathrm{O}=\mathrm{FeO} \text { (in silicate) },+\mathrm{H}_{2}
$$

leads to much higher activities of $\mathrm{FeO}$ than one would have at equilibrium. This tendency for higher $\mathrm{FeO}$ activities increases rapidly with a drop in temperature. Since we impose a constraint on forming solid silicates, our results are for the formation of metastable subcooled liquids and with the liquidus temperatures given above for chondrule type IA and II compositions we can calculate the degree of subcooling needed to obtain compositions close to the average for each of these two chondrule types. Calculated 
compositions of the metastable nebular condensates are given in Table ib at five pressures ranging from 0.001 to 5 atmospheres at temperatures where the $\mathrm{FeO}$ contents of the condensate were close to that of one or of the other of the two chondrule types. We can see that some of the compositions deviate significantly from the measurements. In particular, the results for the low $\mathrm{FeO}$ contents at 5 atmospheres have too much $\mathrm{CaO}$ and $\mathrm{Al}_{2} \mathrm{O}_{3}$ and those at 0.001 atm might be ruled out because of low $\mathrm{Mg} / \mathrm{Si}$ ratios and related differences between the absolute values of the $\mathrm{SiO}_{2}$ and $\mathrm{MgO}$ mole fractions. The other values at pressures between 0.01 through 1 atmosphere are probably within the combined uncertainties in the measurements and calculations. It is likely that somewhat higher pressures (and temperatures) are possible (e.g. 2 atmospheres) and that the pressures might be as low as 0.001 or lower if there are unrecognized uncertainties in e.g., solar abundances or other input data. Thus we see that under conditions defined with well known physical laws (i.e. nucleation theory) we can obtain the compositions of both the type IA and type II chondrules from the same gas phase, in this case from a solar gas. The question on origins transforms to the question: How can these compositions be preserved?

Preservation of Droplet Compositions and Chondrule Formation

These liquids are metastably subcooled below their liquidus temperatures (about $349-582^{\circ} \mathrm{C}$ below for the low FeO type IA material and about $383-579^{\circ} \mathrm{C}$ below for the high FeO type II material). It is well known in pyrometallurgy that liquid silicates catalyze reactions involving solid silicates. This should be especially true where the reactant and/or products are complex chain or network silicates. Thus, nucleation and crystallization of 
the metastable materials in Table $\mathrm{I}$ at or below one of the temperatures listed in Table Ib would lead to their preservation. As has been shown in experimental work ${ }^{25-29}$ many preformed nuclei are necessary to form the porphyritic textures. Consequently, our concept requires a mechanism which would enhance the nucleation rate in individual droplets. It is unfortunate that most of the experiments which have been performed were specifically designed to define constraints for the origin of chondrules by a specific mechanism of reheating solid preformed silicates to partly melt them and then cooling them rapidly. Aside from the difficulty of determining the reheating mechanism which would do this, there is an overwhelming difficulty based on the observations by Hewins ${ }^{25}$ and Hewins and Radomsky ${ }^{26}$ that the porphyritic chondrule textures appear to be formed only when the initial temperatures are somewhat below the liquidus temperature for that composition. When one recalls that most chondrules (about $81 \%$ ) are porphyritic ${ }^{9}$ with a broad range of compositions and hence of liquidus temperatures, this constraint would appear to be impossible to fulfill. For example, we calculate a difference of $122^{\circ}$ between the liquidus temperatures for the average compositions of types IA and II chondrules. Since there is a distribution of compositions within each of these types, the range of liquidus temperatures of individual chondrules is likely to be much higher than $122^{\circ}$. To precisely heat the large majority of droplets to just below the liquidus is highly improbable even in one location, let alone in the many different locations in which the different ordinary chondrites probably formed. As we discuss below, formation of chondrules by reheating precursor materials to somewhat below the liquidus temperatures for a range of different compositions is a natural consequence of the process of recalescence which 
is initiated by crystallization from metastable subcooled liquids such as those we calculated (Table lb). Thus, the probability for fulfilling the reheating condition emphasized by Hewins and Radomsky is far higher for chondrule formation from subcooled liquids than for formation in any other way.

Connolly and Hewins ${ }^{30}$ have shown that the presence of a relatively high density of fine forsteritic dust broadens the range of temperatures where the nucleation of porphyritic chondrules is possible. However, the very presence of dust fines implies that there is an extremely broad and unbelievable rarige of effectiveness of heating different particles if chondrule size objects can be completely melted while fine dust particles, which can be heated far more rapidly, are not melted. Thus the probability of such a dusting process with very rapid and therefore size dependent melting is also unlikely.

Our postulates must fulfill yet another important condition deduced from experiments ${ }^{25,26,28}$ that there be many crystal nuclei present in the largely molten droplets which crystallize to a porphyritic texture. Of course, any mechanism, such as seeding with dust as is presently postulated would also apply for our mechanism if needed. However, within the context of this discussion and of the expected properties and phenomena in a convective nebular gas, there are at least two more plausible ways to generate multiple crystal nuclei. One way stems from the prediction from nucleation theory that the driving force and the rate of nucleation increases the further the temperature drops below (but not too far below) the liquidus temperature whereas, there is a range of temperatures above the glass transition where the rate of crystal growth decreases with decreasing temperature. At a temperature where a droplet is subcooled low enough for the rate of 
nucleation to be large relative to the rate of growth, one might generate a large number of nuclei with very little growth of crystals. The nebula in which this occurs is undoubtedly convective with any part of the nebula decreasing in its time averaged temperature about which the local temperature oscillates during the cyclical compression and expansion stages in the convective motion. Thus, in this mechanism, many nuclei would nucleate during the cooling expansion stage and the nuclei would grow during the heating compression stage after the temperature rises enough. The growing nuclei release their enthalpy of fusion upon crystallization which rapidly raises the temperature above the ambient temperature. Since nucleation and growth is a stochastic process, one, or a very small number of nuclei will initiate the process and then the crystals formed would release enthalpy locally (on a short time scale) and thus sfeed up their own crystallization. This first stage of recalescence in which the temperature rises above the ambient temperature is usually very rapid above the glass transition temperature. As the droplet temperature rises, other nuclei grow, some at the expense of the original crystals. It has been shown that only olivine nucleates under these conditions and continues growing even when the composition is in the pyroxene phase field. The residual glass can be quite high in silica $^{31}$. The temperature rises, not to the liquidus temperature of the total droplet (average about $2026 \mathrm{~K}$ for type IA material, $1904 \mathrm{~K}$ for type II material), but to the lower liquidus of the composition of the droplet after subtracting out the crystallized olivine. Since the low FeO compositions of type IA materials at different pressures are quite close to the binary $\mathrm{MgO}-\mathrm{SiC}_{2}$ system and in the olivine phase field, the phase diagram indicates that the liquidus is a fairly steep function of the amount of crystallized olivine 
and not very much olivine would have to crystallize at the highest feasible pressures (1 atm in Table Ib) to reach the liquidus of the residual liquid. The behavior of the type II materials will be similar with less crystallization from the liquids the higher the gas pressure. The droplet temperature, which is now higher than the ambient temperature, cools by radiation (stage $I I$ of recalescence) at a fairly rapid calculable rate which is likely to be much slower than the recalescence heating. During this stage, more olivine might grow or pyroxene might nucleate. If pyroxene were to nucleate at this stage, the pyroxene (which probably nucleates on the already formed olivine) would partly grow at the expense of the metastable olivine possibly giving the appearance of a relict grain. However, the time scale in stage II is likely to be too brief to either nucleate the pyroxene or to cause the back reaction of the metastable grain. After reaching a temperature close to the ambient temperature the rate of cooling decreases to the probably much slower local cooling (or possibly heating) rate in the nebula (stage III of recalescence). In any case, the ambient temperature would vary cyclically with an overall average temperature drop. It is well known that cyclical ambient temperatures help to catalyze the crystallization (or recrystallization) of solids. It is at this stage where it is most likely that pyroxene would be nucleated and metastable olivines subsumed, at least in part. The product of such a process could be the apparently relict crystals observed in these chondrules. The most metastable olivine grains which are most likely to appear to be relict are the few that initiated stage I (or possibly stage II also) of recalescence, and are most likely to be corroded by the growing pyroxenes which are nucleated later. The process of recalescence thus appears to fulfill the criteria deduced for the production of 
chondrules ${ }^{25-29}$ in that there is a rapid heating, more than one stage of cooling and final crystallization from a temperature below a (calculated) liquidus temperature with some preformed crystal nuclei. The different stages should produce different olivines. For type II chondrules, the initial stage I should produce olivine with a composition of about $\mathrm{Fa}_{21}$; stage II should initially produce a more forsteritic olivine. With further cooling of the chondrule, the compositions produced should evolve to the normal zoning by closed system crystallization, as described by Jones ${ }^{7}$.

Since nucleation is a stochastic process, not all metastable droplets need be nucleated in the foregoing process and the unnucleated droplets would continue to react with the nebula.

Alternatively, one could nucleate the multiple nuclei necessary to form porphyritic textures at stage III of recalescence which was initiated by nucleation by only a small number of nuclei. With cyclical variations in temperature, nucleation rates are greatly increased by a well known mechanism. Thus, many nuclei could be produced in this stage. In addition, with oscillating temperatures, the crystals should ripen, and new phases (including pyroxenes if they haven't formed yet) could nucleate and/or ripen. It is in this stage that the metastabilities resulting from processes in stages I (or possibly) II will be decreased and the easily formed olivines are likely to be transformed to "relicts" and the poikilitic textures produced.

A second class of mechanisms which could lead to porphyritic chondrules depends on external perturbations from the nebula. For example, shock waves, turbulence, and sound waves (e.g. ultra sound in the laboratory) are known to greatly increase crystal 
nucleation rates in liquids. Thus, one of many physical perturbations, which in totality are considered to be very likely to exist in the nebula, (and many of which have been often postulated to explain meteorite properties) could create multiple nuclei in the nebular droplets and initiate recalescene. Since these perturbations are not necessarily uniform, their influence is likely to not be uniform, and not all the droplets need be nucleated at this stage. For example, some of the droplets might be shadowed by other droplets or the energy density of the perturbation may be too small in some places. Such effects, and the stochastic nature of nucleation make it easy to believe that not all droplets need to have crystallized in the first cycle. After nucleation by a nebular perturbation, the course of crystallization and the phenomena should be the same as in the first type of mechanism we discussed above. Either of these two mechanistic possibilities could be repeated thus producing type IA material in one cycle and type II material in a second cycle at a lower temperature.

\section{Discussion}

There are many problems with the currently popular process of chondrule formation by reheating precondensed materials. The paramount problem concerns the need to heat droplets of different compositions to very different temperatures, with temperatures just below their liquidus temperatures needed to form porphyritic textures ${ }^{25}$. Such a process is highly improbable. Hewins and Radomsky find is necessary to postulate that different droplets were heated to different temperatures ranging from $1300-1800^{\circ} \mathrm{C}\left(1400-1750^{\circ} \mathrm{C}\right.$ for $97 \%$ of the droplets) in the same heating event, with the amount of heating being just enough to heat droplets to their liquidus or just below. (Even with nucleation by dust fines, 
another possible mechanism for porphyritic textures, temperatures are limited to just below the melting point of the dust and can not be much higher even with the rapid heating of such small particles. Our mechanism leads to such a range of temperatures below the melting points of the droplets in a natural way by the process of recalescence.

The strong tendencies of silicate droplets to form glasses either partly or completely with rapid cooling without nuclei has been known for a long time $e^{30}$ and the absence of glassy chondrules has to be explained within the context of our postulates. The oscillatory temperatures expected in a nebula are the most likely cause for nucleating crystals in any glass or liquid which escapes the initial chondrule forming stages of recalescence and support for our mechanism requires experiments to define the physical constraints (e.g. temperatures, rates) on such heating-cooling cycles which might produce the observed textures.

So far, we have only discussed the average compositions of types IA and II chondrules. In reality, there is a distribution of compositions which depends on the effective exposed surface/volume ratios of droplets, their velocities relative to the gas, local turbulence and other factors which would make some droplets better collectors of condensates than others. Such relative efficiencies in scavenging of condensing vapor molecules is likely to also be a function of time. One thus expects a range of compositions of porphyritic chondrules from olivine rich to pyroxene rich. Because of the difficulty of nucleating pyroxenes, it is likely that in pyroxene rich porphyries, metastable olivine nucleated first and was the seed for pyroxene which subsumed at least some of the olivine. In any case, if the precursors of the type IA chondrules crystallize and 
effectively cease to react further with the nebula, there will be somewhat more silica in the vapor available for condensation at lower temperatures than was present in our calculations. On the average, this should lead to somewhat lower $\mathrm{Mg} / \mathrm{Si}$ ratios in the uncrystallized droplets (e.g. precursors of types II and III chondrules). In Table I, we see that, indeed, the $\mathrm{Mg} / \mathrm{Si}$ ratio measured in the average type II chondrule (Table la) is lower than all of the calculated ratios (Table lb) for our presumed type II (and type III) precursors. In addition, at yet lower temperatures, the residual silica in the vapor could ultimately lead to even smaller Mg/Si ratios, and pyroxene normative materials with very little, if any, olivine are predicted for the late crystallizing droplets. Thus, droplets which survive to lower temperatures would tend to be relatively high in $\mathrm{FeO}$ and have a relatively small $\mathrm{Mg} / \mathrm{Si}$ ratio. These droplets should resemble the precursors of the type III (radial pyroxene, cryptocrystalline and glassy) chondrules which incidentally also have low liquidus temperatures. The tendency for nucleation of such droplets is low since they are less metastable and in addition are relatively acid and pyroxene normative. Thus it would take a much greater subcooling to nucleate the more easily crystallizable olivine in these drops (partly because the metastable olivine liquidus is at a much lower temperature) than for the type II materials. These droplets would also tend to need external nuclei more than type IA and II materials to initiate their crystallization since olivine is more difficult to nucleate in neutral to acid melts than in basic melts and pyroxene is difficult to nucleate without some seeds (olivine or pyroxene) and enough time and temperature oscillations.

This suggests the mode for crystallizing this material to form excentroradial 
pyroxene chondrules which is consistent with an observation by Hewins and Radomsky ${ }^{26}$ that "Non-porphyritic chondrules (radial pyroxene, glassy and cryptocrystalline), completely melted or superheated, have intermediate to less refractory compositions and lower liquidus temperatures. Similarly, except for a solitary chondrule with the lowest liquidus for porphyries, we see little evidence that chondrules with liquidus temperatures below $1400^{\circ} \mathrm{C}$ were incompletely melted: the least refractory chondrules are not porphyritic." With direct condensation to a metastable liquid, such materials are possible as subcooled liquids or glasses in the pressure range we discuss. Because these acidic melts are difficult to nucleate, in addition to external nuclei, they should undoubtedly require temperatures above the glass transition temperature to nucleate. Thus the glass transition temperatures of these materials imposes an important minimum temperature constraint on their formation within the framework of our concepts. With a knowledge of the glass transition temperatures, we can define a lower limit on the temperature of formation of radial pyroxene chondrules and hence on the nebular pressures where they were formed.

Recent work by Lofgren and Lanier ${ }^{32}$ on the origin of barred olivine chondrules, the only remaining chondrule type, indicates that there is a broad range of conditions, some consistent with our proposed mechanism under which barred olivine chondrules could have been produced. Thus, all the significant types of chondrules could have been formed by crystallization of metastable, subcooled liquid droplets in a solar nebula.

The reader has probably noted that the pressure range in Table $\mathrm{lb}$ is higher than is usually postulated for the solar nebula. The low pressures (and temperatures) which 
are often postulated are based on particular models of the nebula which use measured data on very young (e.g. T-Tauri type) nebulae and lead to pressures which could be as high as $10^{-2}$ at Venus ${ }^{33}$ and higher yet at Mercury. We $e^{34}$ have recently shown that the phase compositions in EL6 chondrites ( $\mathrm{Si}$ in metal, $\mathrm{FeSiO}_{3}$ in enstatite, and albite in plagioclase) is consistent with formation in a high pressure (certainly greater than $10^{-2}$ atmospheres) solar nebula. In the light of earlier calculations ${ }^{3,4}$, we believe that the enstatite chondrites were formed at higher pressures than all the other chondrites and could have been produced closer to the sun and at a higher pressure than Venus. Thus, the possible pressures we obtain in this paper $\left(10^{-2}-1\right.$ atm and possibly $10^{-3}$ to $2 \mathrm{~atm}$.) are consistent with our findings for the enstatite (EL6) chondrites and models for T-Tauri type stars need to be reexamined to see if higher pressures in a nebula can be deduced in a way which is consistent with astronomical observations and with our results. It would be especially important to calculate pressures at radii less than that of Venus.

Our hypotheses remove major problems with current concepts of the formation of chondrules. However, some aspects of the proposed mechanism on nucleation in subcooled liquids (or possibly glasses) needs to be tested for the specific classes of materials in chondrules. Laboratory experiments are necessary to find conditions of temperature and temperature-change where our hypotheses on crystallization are valid. Hopefully, the time scales and rates for the possible validation of our hypotheses are accessible in the laboratory. If correct, our hypotheses will, ultimately, not only explain the textures of chondrules but will also explain their compositions simply.

Up to this point, we have ignored an important fact in Table IA on the alkali 
(sodium) oxide content of porphyritic chondrules (average of 0.45 mole $\% \mathrm{Na}_{2} \mathrm{O}$ in type IA chondrules and 1.51 mole \% in type $\|$ chondrules). Our calculations indicate considerably less alkali oxide in the liquids at all pressures with far less alkali at the lowest pressures then at the highest pressures. There are several possible reasons for this difference. First, is the possibility that the nebular pressures are considerably higher but that segregation of prior $\mathrm{Ca}-\mathrm{Al}$ rich refractory condensates have led to a partial removal of $\mathrm{Ca}$ (and some $\mathrm{Al}$ ) from the gas phase. We don't consider this to be a likely possibility but it is not impossible and would lead to type IA material which is not as high in $\mathrm{Ca}$ and $\mathrm{Al}$ as in e.g. our calculated values at $5 \mathrm{~atm}$. in Table $\mathrm{lb}$ but would have more of the alkalis. The second possibility is that the data on $\mathrm{Na}_{2} \mathrm{O}$ containing materials in our data base is incorrect. Whereas data for $\mathrm{Na}_{2} \mathrm{O}$ containing systems are not as good as for most other materials, they are not likely to be wrong by orders of magnitude. Another effect well known in solid and liquid silicates is a charge compensation effect in which $\mathrm{A}^{3+}$ can substitute for $\mathrm{Si}^{4+}$ by forming an $\mathrm{Al}^{3+}-\mathrm{Na}^{+}$pair thus lowering the activity coefficients for $\mathrm{Na}_{2} \mathrm{O}$. Data for this effect are sparse and do indicate that the activity coefficients of $\mathrm{Na}_{2} \mathrm{O}$ are lowered by this pairing, but that the effect does not seem to be high enough to explain the difference between our equilibrium calculations and the measured levels of $\mathrm{Na}_{2} \mathrm{O}$ in the chondrules. Because more thermodynamic data on the interactions between $\mathrm{Al}^{3+}$ and $\mathrm{Na}^{+}$would be needed to reject this possibility outright, we consider this a possible but unlikely reason for the differences. The most likely possibility is the continued pickup of alkalis by the nebular materials. Since reactions involving chain or crosslinked silicates have been postulated to be kinetically blocked, another mechanism 
is needed which doesn't involve the spherule silicates in a decomposition reaction. An obvious mechanism stems from the fact that even at the temperatures where the type II chondrule like materials in Table Ib were crystallized and frozen in according to our postulates, there is enough residual SiO in the nebular gas to form solid alkali silicates at lower temperatures. The accretion of these later condensing silicates with the chondrules (or their preferred nucleation on chondrule internal and external surfaces) at "metamorphic" temperatures or higher could lead to ion exchange processes between the condensed sodium (or other alkali) bearing silicates and the cations in chondrule silicates. Since monovalent cations are more mobile than divalent cations, they are more likely than other ions to participate in ion exchange reactions with divalent cations in chondrule glasses and even crystals, especially at their surfaces. Temperature cycling in a convecting nebula could speed this process. Indeed, there is evidence even in CAl'S of small crystals of alkali minerals on the surfaces of normal CAI minerals and in interstices which would have to be the result of a secondary emplacement of alkalis from the nebula and some level of reaction or exchange. Alkali minerals (sodalite and/or nepheline) were observed by Fuchs ${ }^{35}$ in all the CAl'S he examined carefully (more than 10), which includes all the CAl'S described in a published paper ${ }^{5}$. Alkali silicates could similarly accrete or form on chondrules and react as best they can with the minerals and glasses in the chondrules.

\section{Summary}

We have developed a model and database for the thermodynamic properties of molten multi-component aluminosilicates. These capabilities have been coupled with a 
database for over 5000 other solid, liquid and gaseous species and phases and a computer program for calculating equilibria, including constrained equilibria, in a complex multicomponent multiphase system. This computer program has been used to calculate the products of condensation from a solar nebula with the imposition of nucleation constraints on the formation of iron alloys and solid silicates. This results in metastable suvcooled liquid aluminosilicates with compositions resembling types IA and type II porphyritic chondrules at two different temperatures at any pressure between $10^{-2}$ and 1 (or possibly $10^{-3}$ to 5 ) atmosphere. If liquid silicates are necessary to catalyze (i.e. flux) the reactions between solid silicates (especially chain and network silicates), then crystallization of the metastable liquids at particular temperatures will largely freeze in the compositions at those temperatures. Crystal nucleation can be spontaneous or externally stimulated by seeding or by nebular perturbations (e.g. shock or sonic waves). Once nucleated, the course of crystallization of the metastable liquids leads to many of the characteristics deduced from experiments as being necessary to form porphyritic chondules. However, more experiments are necessary to deduce possible conditions where these mechanisms are feasible in a convective nebula and to narrow the possible ranges of nebular pressures where these chondrules might have formed. Type Ib and III chondrules can also form readily within the context of our model.

The most important aspects of our results are that (1) the different types of chondrules (types I, II, III) could have been produced from the same gas (and in the same location for any one class of chondrite) and do not need a different gas for each apparent oxidation state and (2) the overwhelming difficulty of current models for making 
porphyritic chondrules by reheating solids of widely different compositions and sizes to just below their (widely different) liquidus temperatures (and also to below the liquidus temperatures of olivine dust) in all the different locations of the different chondrites is not necessary. The initiation of a stage of crystallization just below the liquidus temperature is part of the natural process we propose of crystallization from metastable subcooled liquidus (i.e. recalescence) and does not require an improbable heating mechanism.

\section{Acknowledgements}

The work for this paper by the first author (M.B.) was supported by the U.S. Department of Energy, Division of Materials Sciences, Office of Basic Energy Sciences under contract No. W-31-109-ENG-38. This paper was written while the first author was at the Fach Physikalische Chemie, Philipps Universitat, Marburg, Germany with the generous support of a von Humboldt Preis from the von Humboldt Stiftung. 


\section{$\underline{\text { References }}$}

1. M. Blander and J. L. Katz, Condensation of Primordial Dust, Geochim. Cosmochim. Acta 31, 1025 (1967).

2. M. Blander and M. Abdel-Gawad, The Origin of Meteorites and the Constrained Equilibrium Condensation Theory, Geochim. Cosmochim Acta 33 (6), 701 (1969).

3. M. Blander, The Constrained Equilibrium Theory: Sulfide Phases in Meteorites, Geochim. Comochim. Acta 35, 61-76 (1971).

4. M. Blander, Non-Equilibrium Effects on the Chemistry of Nebular Condensates: Implications for the Planets and Asteroids, ed. T. Gehrels, University of Arizona Press, Tucson (1979) pp. 809-821.

5. M. Blander and L. H. Fuchs, Calcium-Aluminum-Rich Inclusions in the Allende Meteorite: Evidence for a Liquid Origin, Geochim. Cosmochim. Acta 39, 1605-1619 (1975).

6. T. J. McCoy, E. R. D. Scott, R. H. Jones, K. Keil and G. J. Taylor Composition of Chondrule Silicates in LL 3-5 Chondrites and Implications for their Nebular History and Parent Body Metamorphism, Geochim. Cosmochim. Acta 55 601-19 (1991).

7. R. H. Jones, Petrology and Mineralogy of Type II, FeO-Rich Chondrules in Semarkona (LL 3.0): Origin by Closed System Fractional Crystallization with Evidence for Supercooling, Geochim. Cosmochim Acta, 54, 1785-1802 (1990).

8. R. H. Jones and E. R. D. Scott, Petrology and Thermal History of Type IA Chondrules in the Semarkona (LL 3.0) Chondrite, Proc. 19th Lunar and Planetary Science Conf., 523-536 (1989).

9. J. N. Grossman, A. E. Rubin and H. Nagahara, Properties of Chondrules in Meteorites and the Early Solar System, eds. J. F. Kerridge, M: S. Matthews, University of Arizona Press, Tucson, AZ (1988) pps. 619-659.

10. H. Y. McSween Jr., On the Nature and Origin of Isolated Olivine Grains in Carbonaceous Chondrites, Geochim. Cosmochim. Acta 41 411-18 (1977).

11. E. R. D. Scott and G. J. Taylor, Chondrules and Other Components in C, O and E Chondrites: Similarities in their Properties and Origins, Proc. 14th Lunar Planet. Conf. B 275-286 (1983).

12. H. Palme and B. Fegley Jr., High Temperature Condensation of Iron-Rich Olivine in the Solar Nebular, Earth Planet. Sci. Lett., 101 180-195 (1990). 
13. G. Eriksson and K. Hack, ChemSage - A Computer Program for the Calculation of Complex Chemical Equilibria, Metall. Trans., 21 B, 1013-1023 (1990).

14. M. Blander and A. D. Pelton, "Computer-Assisted Analyses of the Thermodynamic Properties of Slags in Coal Combustion Systems," ANLFE-83-19, Argonne National Laboratory, Argonne, IL 60439 (1983).

15. M. Blander and A. D. Pelton, Thermodynamic Analysis of Binary Liquid Silicates and Prediction of Ternary Solution Properties by Modified Quasichemical Equations, Geochim. Cosmochim. Acta 51, 85-95 (1987).

16. A. D. Pelton and M. Blander, Computer Assisted Analyses of the Thermodynamic Properties and Phase Diagrams of Slags, Proc. of the Second Internl. Symposium on Metallurgical Slags and Fluxes, eds. H. A. Fine and D. R. Gaskell, TMS-AIME, Warrendale, PA, pp. 281-294 (1984); M. Blander and A. D. Pelton, ibid, pp. 295304 (1984).

17. A. D. Pelton and M. Blander, Thermodynamic Analysis of Ordered Liquid Solutions by a Modified Quasichemical approach-Application to Silicate Slags, Metall. Trans. 17B, 805-815 (1986).

18. A. Pelton, G. Eriksson and $\mathrm{M}$. Blander, unpublished work incorporating $\mathrm{Al}_{2} \mathrm{O}_{3}$ as a component of multicomponent silicate melts (1991).

19. E. Anders and N. Grevesse, Abundances of the Elements: Meteoritic and Solar, Geochim. Cosmochim. Acta $\underline{53} 197$ (1989).

20. P. Wu, G. Eriksson, A. D. Pelton and M. Blander, Prediction of the Thermodynamic Properties and Phase Diagrams of Silicate Systems: Evaluation of the FeO-MgO$\mathrm{SiO}_{2}$ System, submitted for publication in the ISIJ Journal.

21. R. C. Newton, T. V. Charlu and O. J. Kleppa, Geochim. Cosmochim. Acta 44 933941 (1980).

22. R. G. Berman and T. H. Brown, Heat Capacity of Minerals in the System Sodium Monoxide-Potassium Monoxide-Calcium Oxide-Magnesium Oxide-Iron (II) Oxide, Iron (III) Oxide-AluminiumOxide-SiliconDioxide-TitaniumDioxide-Water-Carbon Dioxide: Representation, Estimation and High Temperature Extrapolation; Contrib. Mineral. Petrol., 89 168-183 (1985); ibid $\underline{94} 252$ (1986).

23. R. G. Berman, T. H. Brown and H. J. Greenwood, An Internally Consistent Thermodynamic Data Base for Minerals in the System Sodium MonoxidePotassium Monoxide-Calcium Oxide-Magnesium Oxide-Iron (II) Oxide-Iron (III) Oxide-Aluminium Oxide-Silicon Dioxide-Titanium Dioxide-Water-Carbon Dioxide, 
Atomic Energy of Canada Ltd., Report TR 377 (1985) 62p.

24 D. J. Frurip and S. H. Bauer, Homogeneous Nucleation in Metal Vapors. 3. Temperature Dependence of the Critical Supersaturation Ratio for Iron Lead and Bismuth, J. Phys. Chem. 81, 1001-6 (1977).

25. R. H. Hewins, Experimental Studies of Chondrules in Meteorites and the Early Solar System, eds. J. F. Kerridge and M. S. Matthews, University of Arizona Press, Tucson, AZ (1988) pps. 660-679.

26. R. H. Hewins and P. M. Radomsky, Temperature Conditions for Chondrule Formation, Meteoritics $\underline{25}$ 309-318 (1990).

27. P. M. Radomsky and R. H. Hewins, Formation Conditions of Pyroxene-Olivine and Magnesian Olivine Chondrules, Geochim. Cosmochim Acta, 3475-3490 (1990).

28. G. E. Lofgren Dynamic Crystallization of Chondrule Melts of Porphyritic and Olivine Composition: Textures Experimental and Natural, Geochim. Cosmochim. Acta $\underline{53}$ $461-70$ (1989).

29. G. E. Lofgren and W. J. Russell, Dynamic Crystallization of Chondrule Melts of Porphyritic and Radial Pyroxene Composition, Geochim. Cosmochim. Acta $\underline{50}$ 1715-26 (1986).

30. H. C. Connolly Jr. and R. H. Hewins, The Production of Chondrule Textures by Introducing Refractory Dust to Superheated Melts (abstr.) Meteoritics 24 354-355 (1991).

31. M. Blander, H. N. Planner, K. Keil, L. S. Nelson and N. L. Richardson, The Origin of Chondrites: Experimental Investigations of Metastable Liquids in the System $\mathrm{Mg}_{2} \mathrm{SiO}_{4}-\mathrm{SiO}_{2}$, Geochim. Cosmochim. Acta 40, 889-896 (1976).

32. G. E. Lofgren and A. B. Lanier, Dynamic Crystallization Study of Barred Olivine Chondrules, Geochim. Cosmochim. Acta $\underline{54}$ 3537-51 (1990).

33. P. Bodenheimer, personal communication (1991).

34. M. Blander, L. Unger, A. Pelton and G. Eriksson, A Possible Origin of EL6 Chondrites in a High Pressure-High Temperature Solar Nebula, in preparation.

35. L. Fuchs, personal communication (1975). 
Table la

Mean Bulk Compositions (Mole \%) and Calculated Liquidus Temperatures $T_{L}(K)$ of 11 Type IA and 11 Type II Porphyritic Chondrules in Semarkona (LL 3.0) After McCoy et al. (1991).

\begin{tabular}{|l|l|l|l|l|l|l|l|l||}
\hline & $\mathrm{T}_{\mathrm{L}}(\mathrm{K})$ & $\mathrm{SiO}_{2}$ & $\mathrm{Al}_{2} \mathrm{O}_{3}$ & $\mathrm{FeO}$ & $\mathrm{MgO}$ & $\mathrm{CaO}$ & $\mathrm{Na}_{2} \mathrm{O}$ & $\begin{array}{l}\mathrm{Mg} / \mathrm{Si} \\
\text { ratio }\end{array}$ \\
\hline $\mathrm{IA}$ & 2026 & 39.64 & 2.03 & 0.89 & 53.68 & 3.32 & 0.45 & 1.354 \\
\hline $\mathrm{II}$ & $1904 \mathrm{~K}$ & 41.19 & 1.45 & 11.38 & 42.61 & 1.86 & 1.51 & 1.034 \\
\hline
\end{tabular}

\begin{tabular}{||l|l|l|l|l|l|l|l||}
\hline \multicolumn{8}{|c|}{$\begin{array}{l}\text { Table Ib } \\
\text { Calculated Bulk Compositions and Temperatures of Metastable Subcooled Liquids } \\
\text { with Compositions Similar to Type IA and Type II Chondrules at Several Pressures }\end{array}$} \\
\hline $\mathrm{T}(\mathrm{K}) / \mathrm{P}(\mathrm{atm})$ & $\mathrm{SiO}_{2}$ & $\mathrm{Al}_{2} \mathrm{O}_{3}$ & $\mathrm{FeO}$ & $\mathrm{MgO}$ & $\mathrm{CaO}$ & $\mathrm{Na}_{2} \mathrm{O}$ & $\begin{array}{l}\mathrm{Mg} / \mathrm{Si} \\
\text { Ratio }\end{array}$ \\
\hline $1791 / 5$ & 37.12 & 7.19 & 0.89 & 46.12 & 8.45 & 0.0018 & 1.242 \\
\hline $1607 / 5$ & 40.37 & 1.22 & 11.37 & 45.22 & 1.42 & 0.015 & 1.120 \\
\hline $1678 / 1$ & 38.22 & 2.68 & 0.89 & 54.82 & 3.16 & 0.0014 & 1.434 \\
\hline $1521 / 1$ & 40.70 & 1.20 & 11.36 & 44.96 & 1.41 & 0.015 & 1.105 \\
\hline $1549 / 0.1$ & 39.97 & 1.85 & 0.89 & 54.97 & 2.18 & 0.0014 & 1.375 \\
\hline $1416 / 0.1$ & 40.95 & 1.19 & 11.38 & 44.78 & 1.40 & 0.017 & 1.094 \\
\hline $1444 / 0.01$ & 41.45 & 1.61 & 0.88 & 54.07 & 1.90 & 0.0017 & 1.304 \\
\hline $1325 / 0.01$ & 41.05 & 1.19 & 11.40 & 44.71 & 1.39 & 0.022 & 1.089 \\
\hline $1353 / 0.001$ & 42.76 & 1.49 & 0.89 & 53.03 & 1.76 & 0.0023 & 1.240 \\
\hline $1246 / 0.001$ & 41.14 & 1.18 & 11.33 & 44.73 & 1.39 & 0.027 & 1.087 \\
\hline
\end{tabular}

We have left out minor constituents such as $\mathrm{TiO}_{2}, \mathrm{Cr}_{2} \mathrm{O}_{3}, \mathrm{MnO}$ and $\mathrm{K}_{2} \mathrm{O}$ which total less than 1 mole \% of the assemblage, and have normalized the remaining constituents to total (within a round-off error) 100 mole \%. 
$1 !$
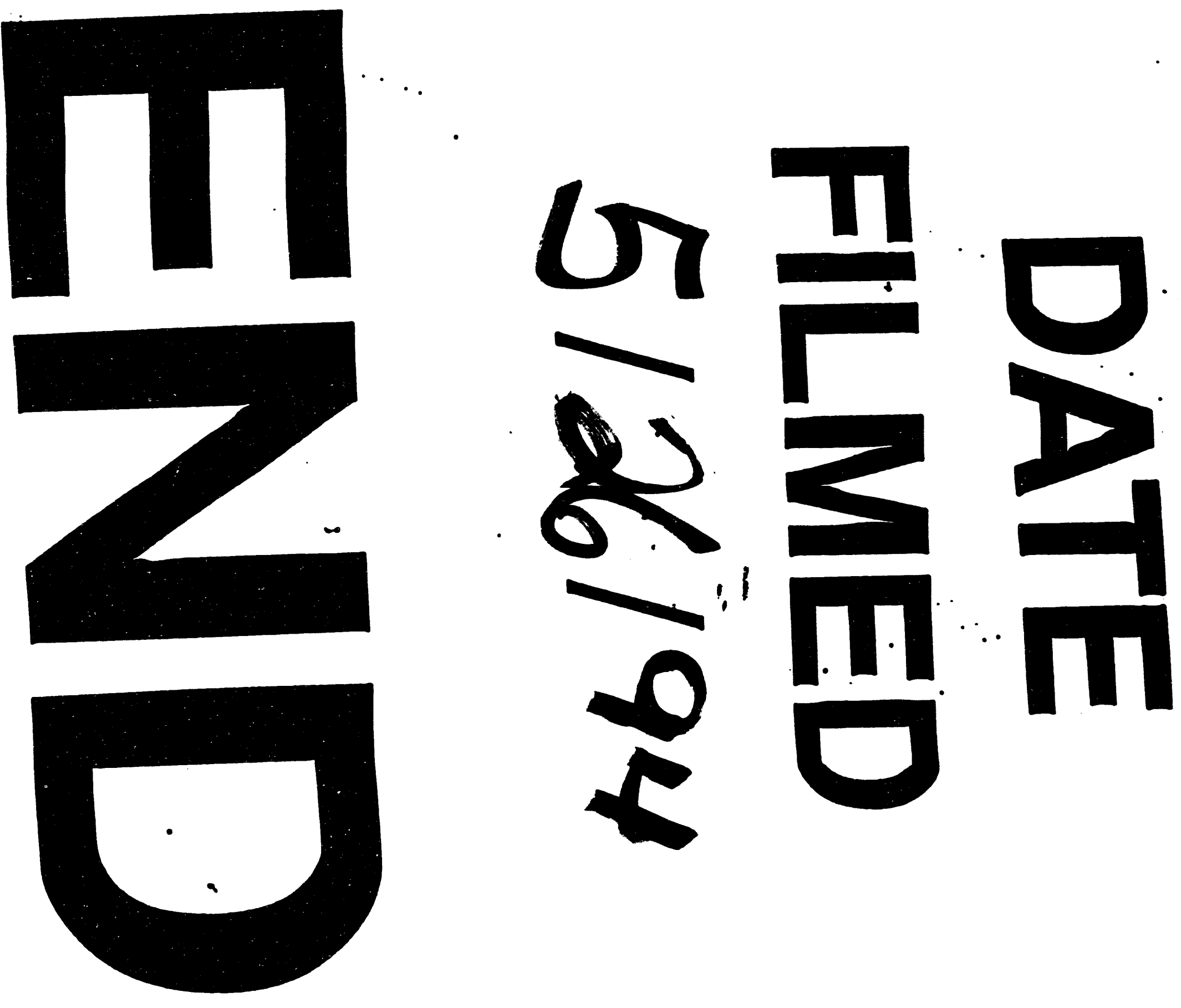


.

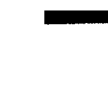

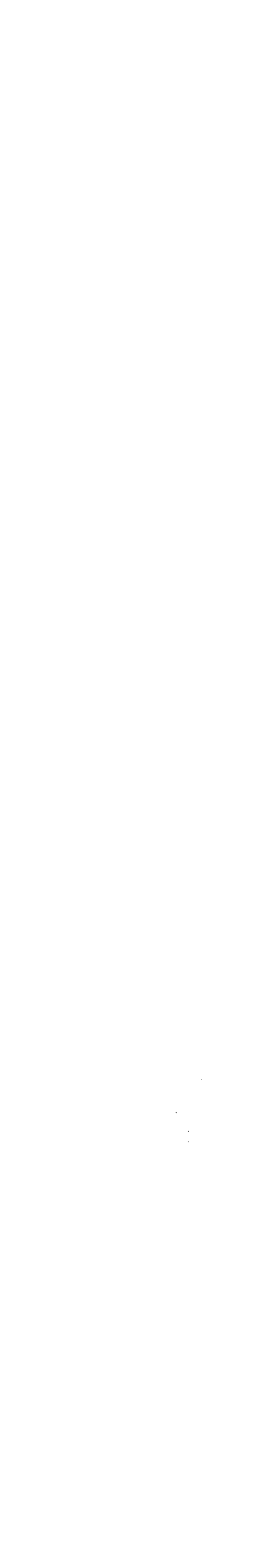

